\title{
BMJ Open Latent tuberculosis infection screening and treatment in congregate settings (TB FREE COREA): protocol for a prospective observational study in Korea
}

\author{
Jinsoo Min (1D , ${ }^{1}$ Hyung Woo Kim, ${ }^{2}$ Helen R Stagg (1) , ${ }^{3}$ Marc Lipman (D) , 4,5,6 \\ Molebogeng X Rangaka, ${ }^{7}$ Jun-Pyo Myong, ${ }^{8}$ Hyeon Woo Yim (D), ${ }^{9}$ Jeong Uk Lim, ${ }^{10}$ \\ Yunhee Lee, ${ }^{8}$ Hyeon-Kyoung Koo, ${ }^{11}$ Sung-Soon Lee, ${ }^{11}$ Jae Seuk Park, ${ }^{12}$ \\ Kyung Sook Cho, ${ }^{13}$ Ju Sang $\mathrm{Kim}^{2}$
}

To cite: Min J, Kim HW, Stagg HR, et al. Latent tuberculosis infection screening and treatment in congregate settings (TB FREE COREA): protocol for a prospective observational study in Korea. BMJ Open 2020;10:e34098. doi:10.1136/ bmjopen-2019-034098

- Prepublication history for this paper is available online. To view these files, please visit the journal online (http://dx.doi. org/10.1136/bmjopen-2019034098).

JM and HWK contributed equally.

Received 05 September 2019 Revised 18 December 2019 Accepted 30 January 2020

Check for updates

(c) Author(s) (or their employer(s)) 2020. Re-use permitted under CC BY-NC. No commercial re-use. See rights and permissions. Published by BMJ.

For numbered affiliations see end of article.

Correspondence to

Dr Ju Sang Kim;

kimjusang@catholic.ac.kr

\section{ABSTRACT}

Introduction South Korea regards tuberculosis (TB) incidence in congregate settings as a serious problem. To this end, systematic latent TB infection (LTBI) diagnosis and treatment were provided to approximately 1.2 million individuals in high-risk congregate settings.

Methods and analysis We designed a prospective cohort study of individuals tested for LTBI, based on the data collected on all persons screened for LTBI as part of the 2017 congregate settings programme in South Korea. Four types of databases are kept: LTBI screening database (personal information and LTBI test results), national health information (NHI) database (socio-demographic data and comorbidities), public healthcare information system (PHIS) database, and the Korean national TB surveillance system database (TB outcomes). Information regarding LTBI treatment at private hospitals and public health centres is collected from NHI and PHIS databases, respectively. The screening data are cleaned, duplicates are removed, and, where appropriate, re-coded to analyse specific exposures and outcomes. The primary objective is to compare the number of active TB cases prevented within 2 years between participants undergoing treatment and not undergoing treatment in the LTBI screening programme in congregate settings. Cascade of care for LTBI diagnosis and treatment will be evaluated among those with a positive LTBI test result. A Cox proportional hazards model will be applied to determine the risk factors for developing active TB.

Ethics and dissemination The protocol is approved by the institutional review boards of Incheon St. Mary's Hospital, the Catholic University of Korea. Study results will be disseminated through peer-reviewed journals and conference presentations.

Trial registration number КСТ0003905

\section{INTRODUCTION}

Approximately one-quarter of the world's population is estimated to have latent tuberculosis infection (LTBI), ${ }^{1}$ which is defined

\section{Strengths and limitations of this study}

- This is a prospective cohort study involving a very large number of people from the general population in an intermediate tuberculosis (TB) burden.

- Long-term follow-up of a large number of participants is possible, and all TB cases can be investigated during the follow-up period because of the unique linkage of electronic databases.

- Data regarding anti-latent TB treatment are indirectly collected based on insurance claims; therefore, treatment adherence cannot be assessed.

- Details of specific clinical data, such as adverse drug reactions and laboratory findings, are not attainable.

- This is an observational study without associated mathematical modelling, thus the impact of the latent TB infection screening programme can only be examined on the individual level, not at the population level through the prevention of transmission.

as a state of persistent immune response to stimulation by Mycobacterium tuberculosis antigens without clinical evidence of active tuberculosis (TB). ${ }^{2}$ Although the treatment of active TB remains a top priority in endemic settings, this approach alone is not sufficient to achieve steep annual reductions in incidence necessary to reach the WHO End TB Strategy targets; thus, prevention of active TB by treatment of LTBI is critical. ${ }^{3}$ Mathematical modelling has shown that protecting $8 \%$ of people with LTBI each year from developing active TB disease could result in a 14-fold decrease in the global incidence of TB in 2050 compared with the incidence in $2013 .{ }^{4}$ WHO recommends that testing and treatment of LTBI should be offered to both adult and child 
contacts of pulmonary TB cases in high-income or upper middle-income countries with an estimated TB incidence rate of less than 100 per 100000 population, such as South Korea. ${ }^{5}$

South Korea has the highest TB incidence and mortality rates among high-income countries. Driven by strong political will, ${ }^{6}$ South Korea has strengthened its TB policy, resulting in a $5.2 \%$ reduction annually in the incidence of newly reported TB cases from 2011 to 2016. Recently, TB outbreaks among health workers in medical institutions and postpartum care centres have become an important social issue, which has raised public awareness and influenced further government commitment. Based on the experience of the Korea Centers for Disease Control and Prevention (KCDC) from 2013 in proactive contact investigations in congregate settings and influenced by the WHO guidelines, ${ }^{5}$ the 'TB Free Korea programme' was started in 2017. As a part of this programme, systematic LTBI testing and preventive TB treatment were provided to approximately 1.2 million individuals in designated high-risk congregate settings.

The introduction of the management of LTBI as a public health intervention requires programme monitoring in order to evaluate quality, effectiveness and impact. ${ }^{7}$ Operational research efforts to enable the effective delivery of such interventions based on settingspecific context and disease epidemiology need to be an integral part of the programmatic management of LTBI. ${ }^{8}$ Further scientific evidence to help understand LTBI is essential to optimise its management and update the guidelines. Here, we hypothesised that systematic LTBI testing and preventive treatment in congregate settings would reduce the national level of active TB incidence. We designed a prospective cohort to evaluate short-term and long-term effects of this project. In this manuscript, we provide an overview of the design, methods and scope of this cohort study.

\section{METHODS AND ANALYSIS}

\section{Aims and objectives}

From 2017, we started recruiting a cohort of people who underwent interferon-gamma release assay (IGRA) testing during the LTBI screening programme in the congregate settings. Our primary objective is to compare the number of active TB cases prevented within 2 years between participants who receive LTBI treatment and those who do not. Other key objectives to be addressed in this cohort study are as follows: (1) to determine the prevalence of LTBI among participants at the initial screening, (2) to determine the prevalence of concurrent active TB among participants at the initial screening, (3) to follow-up with the cohort and identify the incidence of new LTBI cases and (4) to follow-up with the cohort and identify the incidence of new active TB. We also aim to assess the risk factors for both LTBI prevalence and incidence and evaluate outcomes along the cascade of care of LTBI as secondary outcomes. As long-term follow-up is possible with the use of the large interlinked healthcare databases implemented in South Korea, it is possible to examine different outcomes at specific time points (table 1).

\section{Study setting and population}

Our prospective cohort study is based on data systematically collected on all patients within the South Korean LTBI screening programme in congregate settings in 2017. According to the WHO's Global Tuberculosis Report, the incidence of TB was 100 per 100000 population in 2011, which decreased to 77 persons per 100000 population in 2016. However, because of recent stagnation in these declines, the government revised the TB Prevention Act in 2016 to require LTBI screening for employees in highrisk congregate settings. High-risk settings are defined as follows: (1) workers in medical institutions, postpartum care centres, kindergartens, childcare centres, schools and welfare facilities; (2) people in correctional facilities

Table 1 Key objectives of study according to follow-up periods

\begin{tabular}{lll}
\hline Follow-up periods & Key objectives & Secondary outcomes \\
\hline $\begin{array}{l}\text { Short-term } \\
\text { (within 1 year) }\end{array}$ & Prevalence of LTBI & Difference in prevalence of tuberculosis infection among different \\
& & settings \\
& & Risk factor of TB infection \\
& & Distribution of IGRA values \\
& Prevalence of active TB & Cascade of care in LTBI \\
Medium-term & Risk factors of concurrent active TB \\
(within $2 \sim 5$ years) & Incidence of LTBI & Risk factors for developing LTBI \\
& Incidence of active TB & Risk factors for developing active TB \\
& & Efficacy of LTBI treatment on preventing active TB \\
Long-term & Development of drug resistance after LTBI treatment \\
(after 10 years) & Efficacy of LTBI screening & Impact on notification rate for new TB cases in general population \\
& programme at country level & Trend of anti-TB drug resistance (INH and RIF) in general \\
& & population
\end{tabular}

IGRA, interferon-gamma release assay; INH, isoniazid; LTBI, latent TB infection; RIF, rifampin; TB, tuberculosis. 
Owned by Korea Centers for Disease Control and Prevention

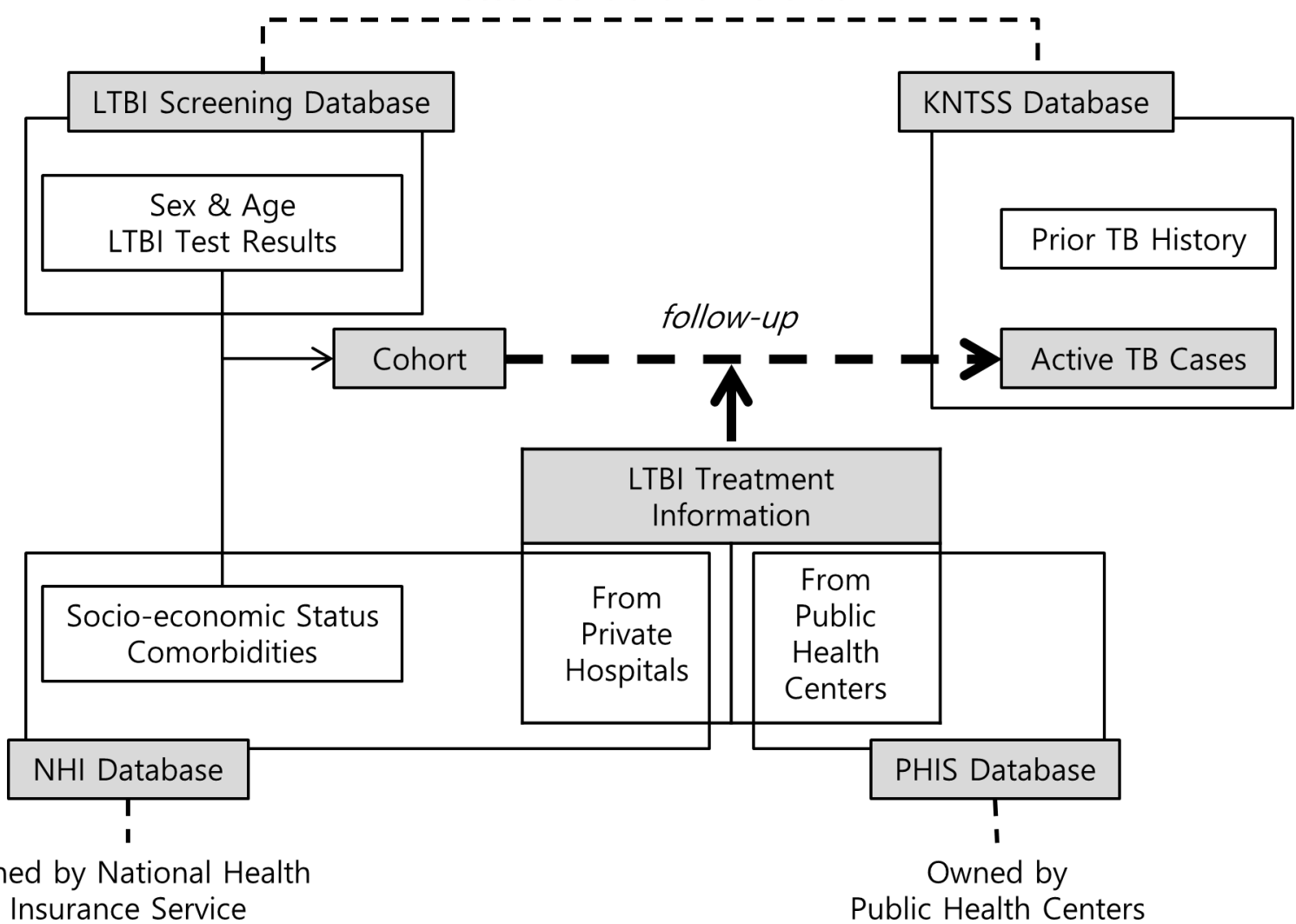

Figure 1 Linkage of databases used in a prospective study of latent tuberculosis infection screening and treatment at congregate settings in South Korea. KNTSS, Korean national TB surveillance system; LTBI, latent TB infection; NHI, national health information; PHIS, public healthcare information system; TB, tuberculosis.

and (3) military conscripts and first-year high school students. The existing employees in these congregate settings were screened in the government programme as of $2017 .^{9}$

\section{Database linkage}

In this study, we will use four databases: the LTBI screening database from the government programme, the national health information (NHI) database, ${ }^{10}$ the public healthcare information system (PHIS) database ${ }^{11}$ and the Korean national TB surveillance system (KNTSS) database (figure 1 and table 2). Anonymised joint keys, which replace the personal identification numbers assigned to the residents of Korea, are used to link the LTBI screening database with three other databases through deterministic matching. This process has been given ethical approval. ${ }^{10}$

KCDC collects data from the participants in the LTBI screening programme and manages the LTBI screening database, which contains personal information (gender, age, types of congregate settings, etc) and the results of IGRA and chest X-rays. Information regarding LTBI treatment (regimen and completion) at private hospitals and public health centres are collected from the NHI and PHIS databases, respectively. Socio-demographic data, such as residential area, insurance types and income level, and comorbidities based on International Classification of Disease-10 (ICD-10) codes are also available in the NHI database established by Korean National Health Insurance Service. At the public health centres, additional information regarding LTBI treatment, such as adverse drug reactions and causes of treatment withdrawal, is collected and stored in the PHIS database. The KNTSS database is a web-based notification system, which receives data regarding all patients who are diagnosed with or treated for TB in South Korea. ${ }^{12}$ The notification data include personal information, microbiological examination results, anti-TB treatment regimens and final treatment outcomes.

\section{Systematic TB screening algorithm}

Participants in the LTBI screening programme were assessed for TB infection according to the Korean national TB guidelines. ${ }^{13}$ After excluding active TB cases based on clinical assessment (previous TB treatment history, contact history, respiratory symptoms related to $\mathrm{TB}$, physical examination) and chest X-ray, LTBI testing was conducted using IGRA. The QuantiFERON-TB Gold In-Tube tests (QGIT, Qiagen, Hilden, Germany) and the interpretation of results were performed according to the manufacturer's instructions. 
Table 2 Sources of database used for the prospective cohort study

\begin{tabular}{|c|c|c|}
\hline Types of database & Information & Ownership \\
\hline LTBI screening database & $\begin{array}{l}\text { Gender and age } \\
\text { Types of occupation } \\
\text { Types of congregate setting } \\
\text { Results of chest X-ray } \\
\text { Results of IGRA }\end{array}$ & $\begin{array}{l}\text { Korea Centers for Disease Control and } \\
\text { Prevention }\end{array}$ \\
\hline National Health Information database & $\begin{array}{l}\text { Comorbidities } \\
\text { Socio-demographic data, including } \\
\text { income level } \\
\text { - LTBI treatment at private hospitals }\end{array}$ & National Health Insurance Service \\
\hline $\begin{array}{l}\text { Public Healthcare Information System } \\
\text { database }\end{array}$ & $\begin{array}{l}\text { LTBI treatment at public health center } \\
\text { Adverse drug reaction after LTBI } \\
\text { treatment } \\
\text { Cause of LTBI treatment withdrawal }\end{array}$ & Public health centres \\
\hline
\end{tabular}

IGRA, interferon-gamma release assay; LTBI, latent TB infection; TB, tuberculosis.

\section{LTBI treatment regimens}

LTBI treatment is offered based on the Korean national TB guidelines, ${ }^{13} 14$ which recommend isoniazid monotherapy for 9 months, rifampin monotherapy for 4 months or isoniazid and rifampin combination therapy for 3 months based on the clinician's decision. Because of the difficulty in communicating effectively with, and delivering information on LTBI concepts to, healthcare professionals, a nationwide education programme was implemented in 2017. ${ }^{6}$ A network with over 300 hospitals was organised for the treatment of LTBI cases identified through the screening programme. Participants could be referred to private hospitals and public health centres for LTBI treatment without restriction.

We will use the NHI and PHIS databases to extract data regarding LTBI treatment at private hospitals and public health centres. We will define individuals as under treatment of LTBI if they have ICD-10 code R76.80 and are prescribed isoniazid, rifampin or a combination of isoniazid and rifampin. Those who started LTBI treatment were categorised into three groups: completion group, withdrawal group and on-treatment group. Individuals are considered to have completed therapy if they are prescribed more than $80 \%$ of total doses within 12 months for isoniazid therapy, 6 months for rifampin therapy or 4 months for isoniazid and rifampin combination therapy. ${ }^{13}$

\section{Definition of active TB cases}

Notification data from the KNTSS database are primarily used in order identify active TB cases. As active TB cases are notified to and monitored by the KNTSS under Korean law, the completeness and timeliness values for TB notification in the KNTSS are high, ${ }^{12}$ assuring its reliability. The NHI database is also used to identify non-notified active TB cases, types of anti-TB drugs and treatment adherence based on insurance claims. The diagnosis of active TB is identified using ICD-10 codes (A15 to A19) and subsequently confirmed by prescriptions for $\geq 3$ anti-TB drugs. The anti-TB drugs include isoniazid, rifampin, ethambutol, pyrazinamide, amikacin, kanamycin, streptomycin, quinolones, ethionamide, cycloserine and para-aminosalicylic acid. The accuracy of identifying active TB cases based on the NHI database was validated and has been used in previous studies on TB. ${ }^{15} 16$ In order to classify previous and active cases, we will identify the notification date of active TB case per individual and examine a temporal relationship between the notification date and the LTBI test date. We will define a new active TB case as one notified more than 30 days after the LTBI test date and a concurrent active TB case as one notified within 30 days of the LTBI test date. If the notification occurred before the LTBI test date, we will consider an individual to have had previous anti-TB treatment. Those with a previous history of anti-TB treatment, who were identified using the KNTSS and NHI databases, will be excluded. Those with active TB notified within 30 days of the LTBI test will also be excluded as we assume that they had subclinical TB infection at the time of screening.

\section{Independent variables}

Factors that might influence the incidence of active TB, such as gender, age, income level, comorbidities or history of close contact with active TB, will be collected and used as independent variables. Income level was described by means of ventiles, with each income ventile consisting of $5 \%$ of the population. Income level was then categorised 
into four groups: low (ventiles 1 to 5 ), lower-middle (6 to 10 ), upper-middle (11 to 15 ) and high (16 to 20 ). Comorbidities were selected based on guidelines published by the WHO, National Institute for Health and Care Excellence and Center for Disease Control and Prevention. ${ }^{17}$ Comorbidities were identified based on ICD-10 codes and claims of procedures and prescribed drugs via Korean electronic data interchange (KEDI) codes, such as HIV infection, organ transplant, silicosis, end-stage renal disease, head and neck cancer, diabetes mellitus, haematological malignancy, those having Tumor Necrosis Factor (TNF) blockers, long-term steroids or chemotherapy, and those with a previous history of gastrectomy.

\section{Analysis of baseline data}

The screening data will be cleaned, duplicates removed and, where appropriate, recoded to analyse specific exposures and outcomes.

To determine the prevalence of LTBI among participants at the initial screening

The primary outcome of prevalent LTBI will be defined as positive results of IGRA among participants at the initial screening.

$$
\begin{aligned}
& \text { prevalence of LTBI = } \\
& \text { no. of participants with a positive IGRA test } \\
& \text { except those with history of TB treatment } \\
& \hline \text { overall no. of participants } \\
& \text { except those with a history of TB treatment }
\end{aligned}
$$

As a secondary outcome, multivariable logistic regression analysis adjusted for independent variables will be used to assess the risk factors for the prevalence of LTBI.

\section{To determine the prevalence of concurrent active TB among} participants at the initial screening

The prevalence of active TB is calculated as the number of concurrent active TB cases divided by the number of all participants, except those with a history of TB treatment.

prevalence of active $\mathrm{TB}=$

no. of participants with concurrent active TB

cases except those with history of TB treatment

overall no. of participants

except those with a history of TB treatment

\section{Analysis of follow-up data \\ Cascade of care in LTBI}

Those with a positive LTBI test result will be followed-up, and the cascade of care for LTBI diagnosis and treatment will be evaluated. ${ }^{18}$ Specific outcomes of interest include the following: (1) the number of people eligible for testing for LTBI; (2) the number who initiated and completed screening with IGRA; (3) the number with positive results with IGRA who had chest X-ray and medical evaluation and (4) the number who were prescribed, started and completed treatment for LTBI. We will also identify the completion rates of LTBI treatment by regimens.

To follow-up the cohort and identify incidence of new LTBI cases Individuals enter the cohort at the date of their LTBI test. In congregate settings such as medical institutions, participants with a negative initial IGRA test result will receive serial IGRA tests annually. The incidence of LTBI will be defined as positive conversion at a later IGRA test and expressed as the number of newly diagnosed LTBI cases per 100000 person-years. The denominator, total persontime at risk, is the sum of total time contributed by those with negative results of initial IGRA test, except those with a history of TB treatment and concurrent active TB cases. If a new LTBI case does not occur, the last date of the serial IGRA test is the final follow-up date.

incidence of LTBI $=\frac{\text { no. with a positive conversion at a later IGRA test }}{\text { total person-time at risk (PY) }}$

When serial follow-up of IGRA test is not performed annually, we will use the midpoint between the latestnegative and earliest-positive test dates as the date of the infection event in order to estimate the incidence. ${ }^{19}$ However, if the testing rate drops below $80 \%$, sensitivity analysis will be conducted.

\section{To follow-up the cohort and identify incidence of new active TB} Individuals enter the cohort at the date of their LTBI test and exit on notification of death or becoming an active TB case. If an active TB case does not occur, the last updated date of the KNTSS database is the final follow-up date. We will follow the individuals in order to identify a new case of active TB after 12, 24 and 60 months of LTBI testing (figure 2). The incidence of active TB is expressed as the number of new active TB cases per 100000 personyears. The denominator, total person-time at risk, is the sum of total time contributed by all participants, except those with a history of $\mathrm{TB}$ treatment and concurrent active TB cases.

$$
\text { incidence of active } \mathrm{TB}=\frac{\text { no. of new active } \mathrm{TB} \text { cases }}{\text { total person-time at risk }(\mathrm{PY})}
$$

Individuals with new active TB will be categorised into four groups based on the IGRA test results and LTBI treatment: (1) those with negative IGRA test results, (2) those with positive IGRA test results who do not start LTBI treatment, (3) those with positive IGRA results who start and complete LTBI treatment and (4) those with positive IGRA results who start but do not complete LTBI treatment. After testing for proportional hazards, a Cox proportional hazards model will be applied to determine the risk factors for developing active TB, if appropriate. Cumulative TB incidence curves will be generated using the Kaplan-Meier method, and differences between groups will be analysed using the log-rank test. The primary outcome is to compare the active TB incidence between treated IGRA-positive participants and 


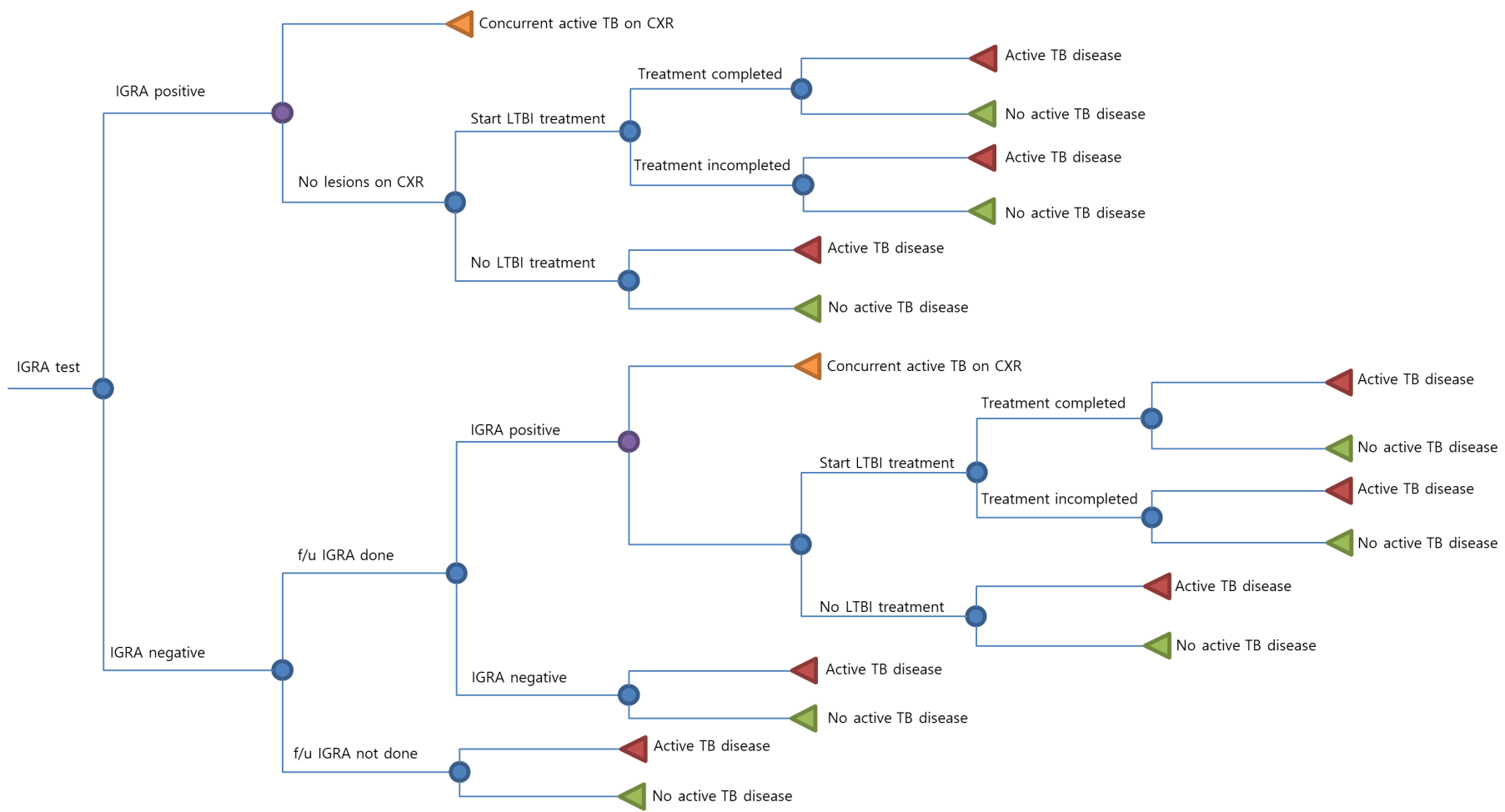

Figure 2 Decision tree model for latent tuberculosis infection screening programme in congregate settings. CXR, chest X-ray; IGRA, interferon-gamma release assay; LTBI, latent TB infection; TB, tuberculosis.

untreated IGRA-positive participants. We will calculate the incidence rate of disease progression in IGRA-positive versus IGRA-negative individuals, separately in the former instance by individuals who did and did not undergo treatment. Among individuals who underwent treatment, figures will also be stratified by treatment regimen. We will calculate positive and negative predictive values of the IGRA test for predicting active TB incidence in the absence of treatment.

\section{Patient and public involvement}

Patients were not involved in setting the research question, the outcome measures, the design or implementation of the study. We plan to translate the results into short, easy-to-read summaries and disseminate it to the relevant patient community through local media.

\section{Ethics and dissemination}

The protocol has been approved for its scientific content and compliance with human subject research regulations by the institutional review boards of Incheon St. Mary's Hospital, the Catholic University of Korea. These committees waived the need for written informed consent from the study participants because this research involves the collection of publicly available and anonymous data. The investigators will disseminate the findings of this research through publication in a peer-reviewed journal and via conference presentations, including key national stakeholders. In compliance with the policy of the International Committee of Medical Journal Editors, this study was registered with the Clinical Research Information Service, Republic of Korea (cris.nih.go.kr) in May of 2019.

\section{DISCUSSION}

The KCDC created a TB epidemic investigation team in 2013, which performs proactive contact investigations in congregate settings at the national level. There have been improvements in the early detection of active TB cases and prevention of its transmission at schools. However, continued TB outbreaks at various congregate settings, such as neonatal intensive care units and postpartum care centres, have become a social issue..$^{20} 21$ As the problem became more prominent, the Korean government has regarded TB incidence in congregate settings as a serious problem and made efforts to it more actively. Thus, mandatory screening for TB infection for workers in facilities (medical institutions, postnatal care centres, kindergartens, childcare centres, schools, welfare facilities and correctional facilities), military conscripts and first-year high school students has been adopted since 2017 as a part of the 'TB free Korea programme'. ${ }^{6}$ Postpartum care centres, kindergarten, childcare centres and schools were selected because of their high risk for TB transmission, especially among infants and children with low immunity. In order to prevent nosocomial TB transmission, healthcare workers at hospitals were also target populations for the screening of TB infection. Military conscripts were selected because of the unique environment of the military units, such as confined living settings and proximity. ${ }^{22}$ First-year high school students were also included because they are at the age when TB cases begin to increase rapidly. To successfully implement the LTBI screening programme in congregate settings and carry out the South Korea LTBI policy, programmatic 
management should include monitoring and evaluation systems. Based on the results of the present study, we expect to provide scientific evidence enabling us to better understand the LTBI burden in South Korea and assess the clinical and epidemiological impact of the LTBI screening programme.

Because of high incidence of nosocomial TB infection among healthcare workers ${ }^{23}$ and delay in isolating active pulmonary TB patients admitted to hospitals ${ }^{24}$ in South Korea, the Korean TB guideline highlights the importance of serial IGRA testing for high-risk healthcare workers and recommend treating those with positive conversion. However, recent studies suggest that serial IGRA testing results in an over-diagnosis of $\mathrm{LTBI}^{25}$ and are not cost-effective ${ }^{26}$ in the North American setting. We plan to conduct sensitivity analysis using the different cut-offs for conversion or confirmatory tests, which were suggested to mitigate the over-diagnosis of LTBI.

Korea underwent short-term but intense socioeconomic changes after liberation from the Japanese occupation during World War II. In parallel with economic prosperity, South Korea achieved admirable control of TB in the past half century. ${ }^{27}$ Based on this rapid change in the socioeconomic status in South Korea, age-period-cohort modelling can clarify the relative and independent effects of influential exposures shared by each birth cohort and influential exposures experienced by all birth cohorts on TB incidence over time. ${ }^{28} 29$ As long-term follow-up of our cohort is feasible, we can identify biological, historical and socioeconomic determinants in long-term trends of TB status and provide evidence for designing effective TB policies and public health interventions based on ageperiod-cohort analysis.

There are several key strengths of the current 'TB FREE COREA' study. First, it is a cohort study involving a very large number of people from the general population. Previous work has been undertaken in mainly immunecompromised high-risk groups or small hospital-based cohorts. ${ }^{30}$ The outcomes of our study can offer rich insights into the intermediate-risk or low-risk groups of LTBI within an intermediate TB-burden country, such as South Korea. Second, it is a prospective study designed by a multidisciplinary team of clinicians, epidemiologists, statisticians and other related experts. When designing the study protocol, we set specific goals and selected several key questions in order to plan data collection suitable for each key question. Third, an active TB case is a nationally notifiable disease and is monitored by the KNTSS. All the cases diagnosed and treated under the national health insurance system are recorded in the NHI database. Thus, using data-linkage between the unique electronic databases KNTSS and NHI, all TB cases can be scrutinised during the follow-up period, thereby minimising the loss of detecting new cases. Fourth, long-term follow-up of a large number of participants is possible because of the unique electronic database of the NHI. This database has been extensively used to establish basic platforms for customised retrospective cohort data. ${ }^{10}$
A periodic monitoring and evaluation process will be undertaken by the advisory boards.

Despite these strengths, the major limitation of the present study is that we receive only a limited data set of clinical, social and demographic information. The details regarding adverse drug reactions during the preventive therapy are not collected, which are major concerns of LTBI treatment. It is also not possible to collect specimens from individuals for genotyping tests, which are important to understand the dynamic of TB transmission. Further clinical prospective cohort studies will be necessary in order to address these LTBI research gaps. Second, completeness of an LTBI treatment is defined by the number of prescribed anti-TB drugs based on insurance claims. Since adherence to the treatment cannot be assessed during the follow-up period, we hypothesised that all prescribed anti-TB drugs are taken.

The role of LTBI treatment on reducing TB incidence at the national level depends on the country's TB epidemiology. Although the current national initiative which aims to screen and treat LTBI in congregate settings is conducted in South Korea, scientific evidence for its effectiveness is lacking and its public health impact is still unknown. The project was carried out under South Korea's strong political will to eliminate $\mathrm{TB}$ disease along with the adoption of the End TB Strategy. ${ }^{5}$ The WHO recently issued updated and consolidated guidelines for people with LTBI, aiming to expand testing and improve treatment; it highlighted LTBI as a key component of a comprehensive control strategy in both high and low prevalence settings. ${ }^{17}$ South Korea is the first country to roll out the LTBI screening programme for the general population. The result of this cohort study will identify most vulnerable populations with LTBI who will progress to active TB and help KCDC develop public health policies towards them. It will also contribute towards highlighting the importance of the LTBI programme as a key component of WHO's End TB Strategy.

\section{Author affiliations}

${ }^{1}$ Division of Pulmonary and Critical Care Medicine, Department of Internal Medicine, Daejeon St. Mary's Hospital, College of Medicine, The Catholic University of Korea, Seoul, Korea (the Republic of)

${ }^{2}$ Division of Pulmonary and Critical Care Medicine, Department of Internal Medicine, Incheon St. Mary's Hospital, College of Medicine, The Catholic University of Korea, Seoul, Republic of Korea

${ }^{3}$ Usher Institute of Population Health Sciences and Informatics, The University of Edinburgh, Edinburgh, United Kingdom

${ }^{4}$ Royal Free London NHS Foundation Trust, London, United Kingdom

${ }^{5}$ UCL-TB, University College London, London, United Kingdom

${ }^{6}$ UCL Respiratory, Division of Medicine, University College London, London, United Kingdom

${ }^{7}$ Institute for Global Health, University College London, London, United Kingdom ${ }^{8}$ Department of Occupational and Environmental Medicine, Seoul St. Mary's Hospital, College of Medicine, The Catholic University of Korea, Seoul, Korea (the Republic of)

${ }^{9}$ Department of Preventive Medicine, College of Medicine, The Catholic University of Korea, Seoul, Korea (the Republic of)

${ }^{10}$ Division of Pulmonary and Critical Care Medicine, Department of Internal Medicine, Seoul St. Mary's Hospital, College of Medicine, The Catholic University of 
Korea, Seoul, Korea (the Republic of)

${ }^{11}$ Division of Pulmonary and Critical Care Medicine, Department of Internal Medicine, Ilsan Paik Hospital, Inje University College of Medicine, Goyang, Korea (the Republic of)

${ }^{12}$ Division of Pulmonary Medicine, Department of Internal Medicine, Dankook University College of Medicine, Cheonan, Chungcheongnam-do, Korea (the Republic of)

${ }^{13}$ Division of Social Service Projects, Office for Social Welfare Policy, Korea Ministry of Health and Welfare, Sejong, Sejong, Korea (the Republic of)

\section{Twitter Helen R Stagg @hr_stagg}

Contributors Study design: JM, HWK, JP-M, HWY, JUL, YL, KHK, LSS, PSJ, KSC and JSK. Funding acquisition: JSK. Manuscript drafting: JM, HWK and JSK. Critical manuscript review: HRS, ML, MXR, JP-M, HWY, SSL, PJS, KSC and JSK. All authors read and approved the final manuscript.

Funding This work was supported by the Research Programme funded by the Korea Centers for Disease Control and Prevention (2019E310200). The funder had no role in study design, conduct, data analysis and interpretation or preparation of the manuscript. This work was approved and had undergone peer-reviewed by the funding body.

Competing interests None declared.

Patient consent for publication Not required.

Ethics approval Institutional Review Boards of Incheon St. Mary's Hospital, the Catholic University of Korea (IRB no. OC19ZESE0023).

Provenance and peer review Not commissioned; externally peer reviewed.

Open access This is an open access article distributed in accordance with the Creative Commons Attribution Non Commercial (CC BY-NC 4.0) license, which permits others to distribute, remix, adapt, build upon this work non-commercially, and license their derivative works on different terms, provided the original work is properly cited, appropriate credit is given, any changes made indicated, and the use is non-commercial. See: http://creativecommons.org/licenses/by-nc/4.0/.

\section{ORCID iDs}

Jinsoo Min http://orcid.org/0000-0001-6091-518X

Helen R Stagg http://orcid.org/0000-0003-4022-3447

Marc Lipman http://orcid.org/0000-0001-7501-4448

Hyeon Woo Yim http://orcid.org/0000-0002-3646-8161

\section{REFERENCES}

1 Houben RMGJ, Dodd PJ. The global burden of latent tuberculosis infection: a re-estimation using mathematical modelling. PLoS Med 2016;13:e1002152.

2 Fox GJ, Dobler CC, Marais BJ, et al. Preventive therapy for latent tuberculosis infection-the promise and the challenges. Int $\mathrm{J}$ Infect Dis 2017;56:68-76.

3 Uplekar M, Weil D, Lonnroth K, et al. Who's new end TB strategy. The Lancet 2015;385:1799-801.

4 Dye C, Glaziou P, Floyd K, et al. Prospects for tuberculosis elimination. Annu Rev Public Health 2013;34:271-86.

5 Guidelines on the management of latent tuberculosis infection: World Health organization 2015.

6 Go U, Park M, Kim U-N, et al. Tuberculosis prevention and care in Korea: evolution of policy and practice. J Clin Tuberc Other Mycobact Dis 2018;11:28-36.
7 Getahun H, Matteelli A, Abubakar I, et al. Management of latent Mycobacterium tuberculosis infection: WHO guidelines for low tuberculosis burden countries. Eur Respir J 2015;46:1563-76.

8 Getahun H, Matteelli A, Abubakar I, et al. Advancing global programmatic management of latent tuberculosis infection for at risk populations. Eur Respir J 2016;47:1327-30.

9 Cho KS, Park WS, Jeong HR et al. Prevalence of latent tuberculosis infection at congregated settings in the Republic of Korea, 2017. Public Health Weekly Report 2018;11:348-54.

10 Cheol Seong S, Kim Y-Y, Khang Y-H, et al. Data resource profile: the National health information database of the National health insurance service in South Korea. Int J Epidemiol 2017;46:799-800.

11 Ryu S, Park M, Lee J, et al. Web-Based integrated public healthcare information system of Korea: development and performance. Healthc Inform Res 2013;19:314-23.

12 Kang H-Y, Yoo H, Park W, et al. Tuberculosis notification completeness and timeliness in the Republic of Korea during 20122014. Osong Public Health Res Perspect 2016;7:320-6.

13 Korean guidelines for tuberculosis 3rd edition. Osong, South Korea: joint Committee for the revision of Korean guidelines for tuberculosis, and Korea centers for disease control and prevention 2017.

14 Min J, Kim JS. Diagnosis and treatment of latent tuberculosis infection. J Korean Med Assoc 2019;62:11.

15 Lee C-H, Kim K, Hyun MK, et al. Use of inhaled corticosteroids and the risk of tuberculosis. Thorax 2013;68:1105-13.

16 Min J, Kwon SK, Jeong HW, et al. End-Stage renal disease and risk of active tuberculosis: a nationwide population-based cohort study. $J$ Korean Med Sci 2018;33:e341.

$17 \mathrm{Kim} \mathrm{HW}$, Kim JS. Treatment of latent tuberculosis infection and its clinical efficacy. Tuberc Respir Dis 2018;81:6-12.

18 Alsdurf H, Hill PC, Matteelli A, et al. The cascade of care in diagnosis and treatment of latent tuberculosis infection: a systematic review and meta-analysis. Lancet Infect Dis 2016;16:1269-78.

19 Vandormael A, Dobra A, Bärnighausen T, et al. Incidence rate estimation, periodic testing and the limitations of the mid-point imputation approach. Int J Epidemiol 2018;47:236-45.

20 Han S, Lee S, Park M. Tuberculosis epidemic investigations in congregate settings, 2016. Public Health Weekly Report 2017;10:1116-20.

21 Ahn JG, Kim DS, Kim KH. Nosocomial exposure to active pulmonary tuberculosis in a neonatal intensive care unit. Am J Infect Control 2015;43:1292-5.

22 C-g Y, Kang DY, Jung J, et al. The infectivity of pulmonary tuberculosis in Korean army units: evidence from outbreak investigations. Tuber Respir Dis 2019;82:298-305.

23 Park Y, Kim SY, Kim JW, et al. Serial testing of healthcare workers for latent tuberculosis infection and long-term follow up for development of active tuberculosis. PLoS One 2018;13:e0204035.

$24 \mathrm{Heo} \mathrm{DH}$, Seo JW, Kim JH, et al. Delays in isolating patients admitted to hospital with pulmonary tuberculosis in Korea. J Korean Med Sci 2019;34:e270.

25 Moses MW, Zwerling A, Cattamanchi A, et al. Serial testing for latent tuberculosis using QuantiFERON-TB gold in-tube: a Markov model. Sci Rep 2016;6:30781.

26 Mullie GA, Schwartzman K, Zwerling A, et al. Revisiting annual screening for latent tuberculosis infection in healthcare workers: a cost-effectiveness analysis. BMC Med 2017;15:104.

$27 \mathrm{Kim} \mathrm{JH}$, Yim J-J. Achievements in and challenges of tuberculosis control in South Korea. Emerg Infect Dis 2015;21:1913-20.

$28 \mathrm{Heo}$ J, Jeon S-Y, Oh C-M, et al. The unrealized potential: cohort effects and age-period-cohort analysis. Epidemiol Health 2017;39:e2017056.

29 Iqbal SA, Winston CA, Bardenheier BH, et al. Age-Period-Cohort analyses of tuberculosis incidence rates by Nativity, United States, 1996-2016. Am J Public Health 2018;108:S315-20.

30 Park JS. The prevalence and risk factors of latent tuberculosis infection among health care workers working in a tertiary hospital in South Korea. Tuberc Respir Dis 2018;81:274-80. 